Musées, Patrimoine et Culture scientifiques et techniques

$146 \mid 2013$

mars-avril 2013

\title{
Les effets de la composition spectrale des sources électriques sur la conservation des objets du patrimoine
}

Jean-Jacques Ezrati

\section{OpenEdition}

Journals

Édition électronique

URL : http://journals.openedition.org/ocim/1193

DOI : 10.4000/ocim. 1193

ISSN : 2108-646X

Éditeur

OCIM

Édition imprimée

Date de publication : 1 mars 2013

Pagination : 5-11

ISSN : 0994-1908

\section{Référence électronique}

Jean-Jacques Ezrati, «Les effets de la composition spectrale des sources électriques sur la

conservation des objets du patrimoine », La Lettre de I'OCIM [En ligne], 146 | 2013, mis en ligne le 01

mars 2015, consulté le 19 avril 2019. URL : http://journals.openedition.org/ocim/1193 ; DOI :

10.4000/ocim. 1193 


\title{
Les effets de la composition spectrale des sources électriques sur la conservation des objets du patrimoine
}

\author{
Jean-Jacques Ezrati *
}

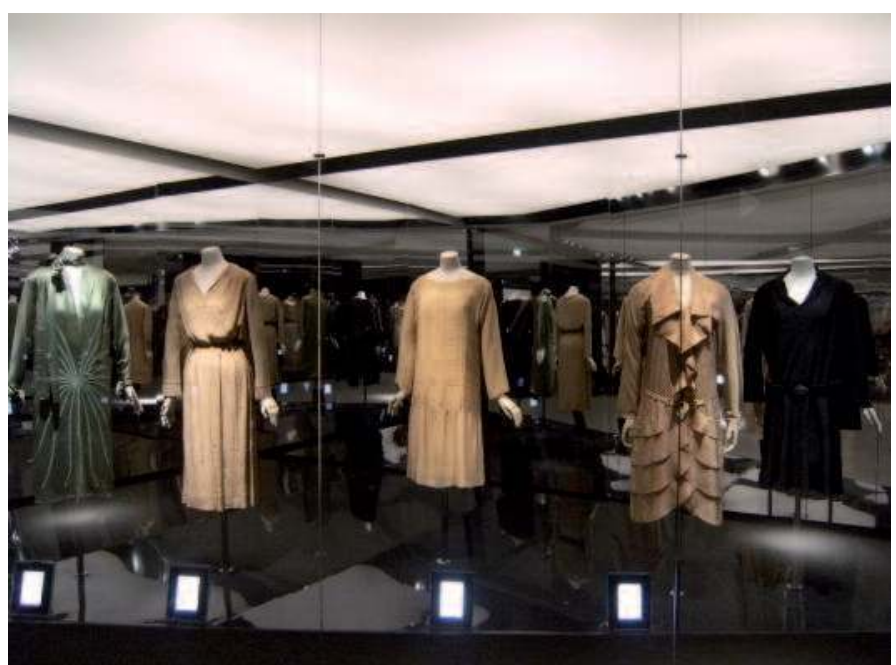

L'éclairage de l'exposition Madeleine Vionnet, créatrice de mode, présentée au musée des Arts décoratifs en 2010, devait composer avec la grande fragilité des objets présentés. (๑) J.J. Ezrati

* Jean-Jacques Ezrati est éclairagiste conseil jean-jacques.ezrati@wanadoo.fr
Spécialiste reconnu des problèmes

d'éclairage et de lumière dans les institutions muséales, l'auteur livre les résultats de deux expérimentations et d'une application pratique mettant bien en évidence que la dégradation observée sur les objets exposés dans les musées est principalement due à l'action du rayonnement visible et non aux seuls ultraviolets.

Lorsque j’ai commencé mon activité comme éclairagiste conseil à la direction des musées de France, ma pratique sur les causes de dégradation des objets du patrimoine était des plus sommaires. J'ai été aidé dans ma tâche essentiellement par deux personnalités, Gaël de Guichen de l'ICCROM et Jean Lapeyre, conservateur à l'Inspection des musées classés et contrôlés, ainsi que par deux ouvrages l'un de Brill (1), l'autre de Garry Thomson (2). Puis avec Marie-Odile Kleist nous avons monté la Cellule de conservation scientifique et technique (3). Pour tous les acteurs de la conservation, il était évident que les causes de dégradation étaient liées à la nature des matériaux, à la composition spectrale des sources et à l'exposition lumineuse. À cette époque, dans les années 1980, nous étions en France, à part quelques exceptions, très peu sensibilisés à ces données ; il fallait donc les diffuser au maximum, notamment le fait que plus les rayonnements de faibles longueurs d'ondes prédominent, en particulier les ultraviolets, plus le danger est grand.

Toujours, dans les mêmes années, j'ai rencontré Robert Julien qui venait de créer l'OCIM et son support La Lettre de l'OCIM. C'est dans le numéro 2 que j’ai signé mon premier article sur le sujet ${ }^{(4)}$. Trente ans 
plus tard, bien que tout ce qui concerne la lumière du jour et la protection contre les ultraviolets soit toujours valable, il y a lieu - suite à mes dernières expérimentations au Centre de Recherche et de Restauration des Musées de France (C2RMF) - d'être un peu plus pragmatique sur la nécessité d'une protection vis-àvis des sources artificielles pour investir au mieux les faibles moyens mis à disposition pour la conservation préventive.

\section{L'élimination du rayonnement UV des sources fluorescentes}

\section{Pertinence d'une étude}

On nous a appris, et nous l'avons toujours enseigné, que les rayonnements optiques des sources de lumière artificielles, en particulier les tubes fluorescents, représentent pour les objets du patrimoine un danger potentiel pour la conservation de ces derniers. À cet effet les recommandations tant nationales qu'internationales exigent la suppression des rayonnements les plus nocifs tels les rayonnements ultraviolets et infrarouges ${ }^{(5)}$.

Nous savons que l'action des ultraviolets résulte d'effets photochimiques, notamment sur les objets d'origines organiques, en agissant sur les liaisons CarboneSodium, Carbone-Oxygène, Carbone-Carbone, et Carbone-Hydrogène. Si un bon filtre contre les ultraviolets est un filtre auquel correspond un filtrage parfait à $320 \mathrm{~nm}(0 \%)$ et un filtrage réduit à $50 \%$ à $400 \mathrm{~nm}$, de la valeur transmise dans le visible à $550 \mathrm{~nm}$, estce suffisant comme moyen de protection ? De plus y a-t-il augmentation du danger avec une plus grande proportion de rayonnements de courtes longueurs d'onde, donc pour les sources de température de couleur supérieure à $5000 \mathrm{~K}$, l'étude qui suit tente de le vérifier (6).

\section{Le protocole d'étude}

Pour la réalisation de cette étude nous avons choisi deux tubes fluorescents en usage dans les musées, conseillés pour leur excellent rendu des couleurs, avec un grand écart de température de couleurs entre elles, soit l'un d'une température de couleur de $2700 \mathrm{~K}$ (tube fluorescent 927) et l'autre de $6500 \mathrm{~K}$ (tube fluorescent 965).

Comme élément test, pour quantifier les effets de décoloration, nous avons opté pour les échantillons numéros 1, 2 et 3 de "l'échelle de la laine bleue » $(7)$, test internationalement reconnu pour classer la solidité à la lumière des textiles et très souvent utilisé en conservation préventive dans un but similaire.
De chaque numéro d'échantillon nous avons réalisé trois éprouvettes : la première protégée par un filtre anti UV (LEE 226), la seconde par un filtre clair (LEE 130) pour obtenir un éclairement identique dû à l'absorption dans le visible, et enfin un dernier comme référent sous une protection opaque. Les deux premières éprouvettes placées à quelques centimètres des tubes ont reçu chacune un éclairement de 5000 lux, ce qui correspond à une exposition lumineuse durant 150 heures de $750 \mathrm{Klx} / \mathrm{h}$. Toutes les 10 heures nous avons effectué une mesure de la couleur, dans l'espace CIELAB ${ }^{(8)}$ de chaque éprouvette exposée et avons calculé la différence de coloration, c'est-à-dire les écarts de couleur DE $\mathrm{a}^{*} \mathrm{~b}^{*}{ }^{(9)}$ en fonction de l'éprouvette en réserve afin d'obtenir les graphes ci-dessous :

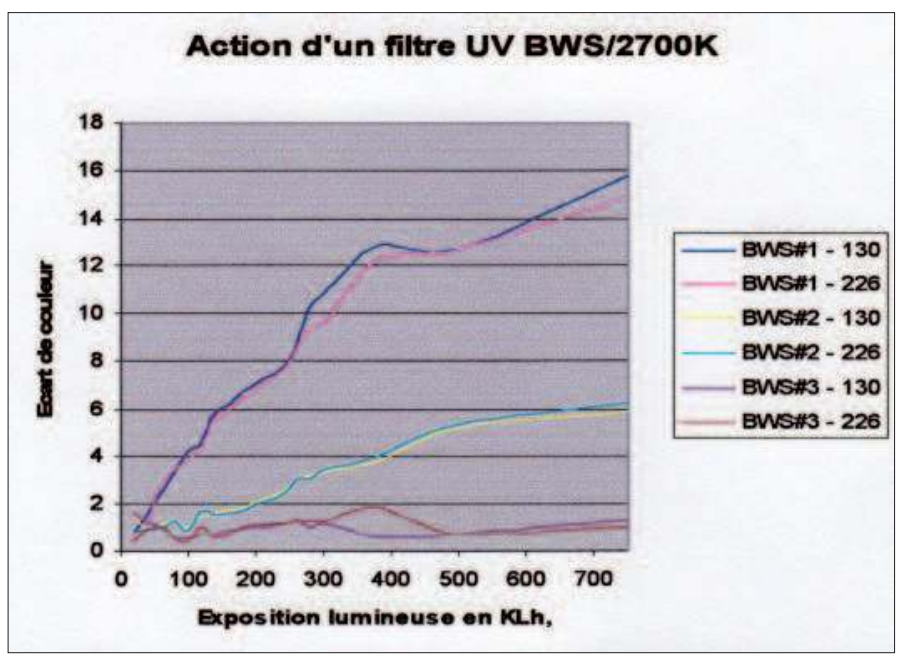

Comparaison de l'action d'un filtre anti UV sur un tube fluorescent type 927

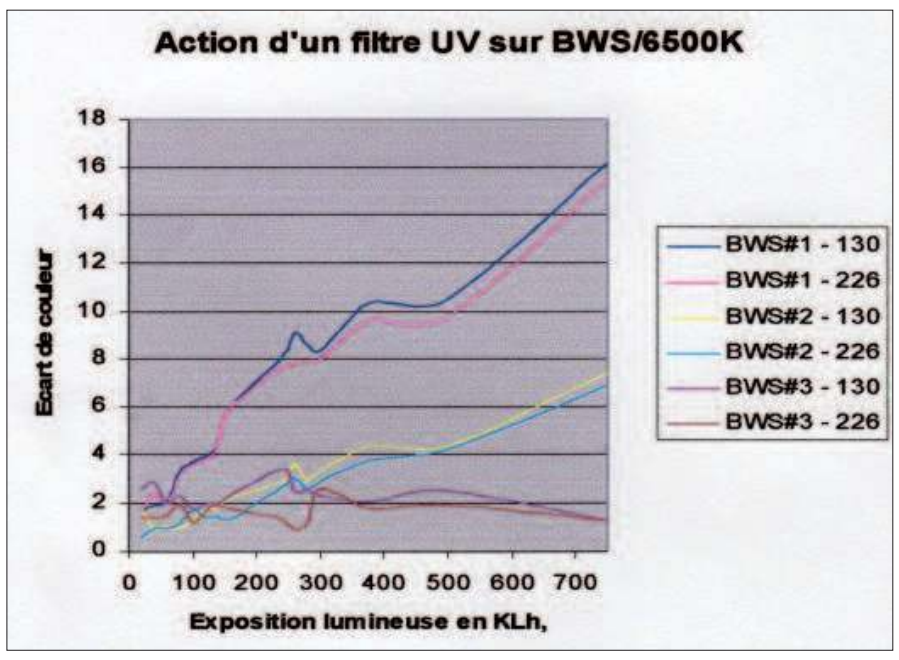

Comparaison de l'action d'un filtre anti UV sur un tube fluorescent type 965 


\section{Analyse des résultats}

On constate que, dans le premier cas (tube fluorescent de température de couleur de $2700 \mathrm{~K}$ ), pour une exposition lumineuse de $500 \mathrm{Klux} / \mathrm{h}$ (soit un éclairement de 50 lux pendant 3,5 ans) de l'échantillon BWS\#1, le filtrage des UV n'a aucune influence, la dégradation est identique (il en est de même pour des expositions lumineuses plus faibles). Avec les échantillons BWS\#2 et BWS\#3 la situation est tout aussi semblable. On en déduit que la quantité d'ultraviolet émise par la source de lumière étant très faible, son action sur la dégradation est marginale. Par contre pour l'échantillon BWS\#1, la dégradation est belle et bien visible $(\mathrm{D}=4)$ dès $100 \mathrm{Kl} x / \mathrm{h}$ (soit moins d'un an d'exposition à 50 lux), que cela soit avec ou sans protection. Pour un objet moins sensible (BWS\#2), cette décoloration n'adviendra qu'après plus de $400 \mathrm{Kl} x / \mathrm{h}$ soit 2,5 ans d'exposition à 50 lux.

Sous le tube fluorescent de $6500 \mathrm{~K}$ de température de couleur on retrouve une tendance similaire, néanmoins on peut remarquer qu'après une certaine dégradation $(\mathrm{DE}>8)$ l'effet de la protection du filtre anti ultraviolet se fait légèrement remarquer sur le graphe mais visuellement n'a aucun effet sur l'apparence.

\section{Comparaison des effets d'autres sources lumineuses d'éclairage localisé sur la dégradation des objets de collection}

\section{Pertinence}

$\mathrm{Si}$ les tubes fluorescents équipent les luminaires d'éclairage général ainsi que des luminaires à optique asymétrique pour un éclairage localisé dirigé, appelé « en lèche-mur », les projecteurs pour l'éclairage localisé qu'il soit focalisé ou cadré, utilisent d'autres types de sources.

En grande majorité on trouvera, encore aujourd'hui, des lampes halogènes, encore quelques fois des sources aux halogénures métalliques mais de plus en plus des diodes électroluminescentes (LEDs). Parmi les arguments employés par les fabricants de ces dernières, le fait qu'elles n'émettent pas de rayonnement ultraviolet, leur fait dire que c'est un atout majeur pour la conservation. Qu'en est-il vraiment ? Pour le savoir le plus simple est donc de comparer à éclairement égal, ou mieux dose égale, les effets de cette source par rapport aux autres sources conventionnelles.

\section{Le protocole d'étude}

Pour être dans des conditions de températures de couleurs les plus proches les unes des autres, la lampe halogène est un modèle à $4100 \mathrm{~K}$ (Solux), la lampe aux halogénures métalliques a une température de couleur légèrement inférieure et la diode électroluminescente affiche une température de couleur de $4100 \mathrm{~K}$.

Comme pour l'étude précédente nous avons utilisé « l'échelle de la laine bleue » et découpé quatre échantillons du test $\mathrm{n}^{\circ} 1$ (BWS\#1) dont la sensibilité est la plus grande. Trois échantillons ont servi au test, un pour chaque source, le quatrième, mis en réserve. Suivant le même protocole de mesure nous avons effectué les mesures afin de calculer l'écart de couleur en fonction de l'exposition lumineuse. L'éclairement mesuré au droit de chaque élément test est de 10000 lux. Au bout de trente heures de fonctionnement, l'exposition lumineuse correspondante est donc $300000 \mathrm{~lx} / \mathrm{h}$ ce qui correspond, par exemple, à un éclairement de 100 lux en continue tout le long d'une année.

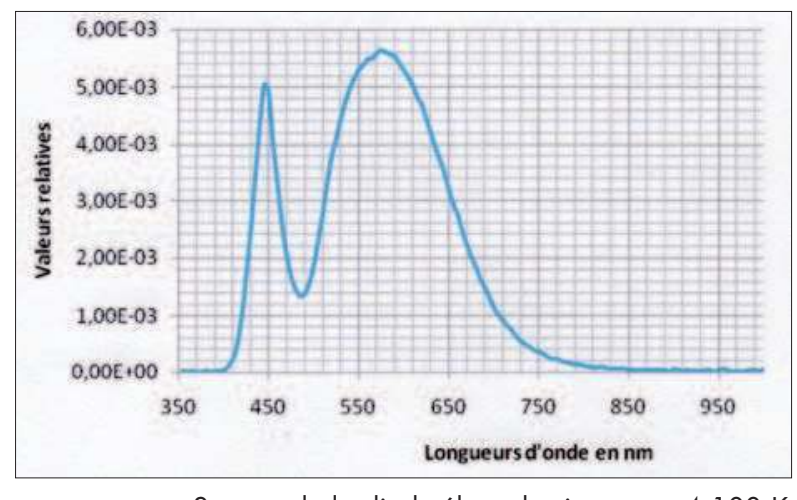

Spectre de la diode électroluminescente $4100 \mathrm{~K}$

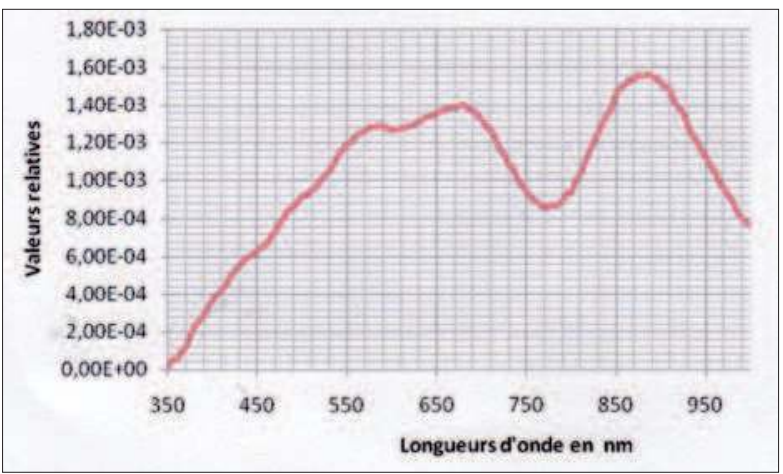

Spectre de la lampe halogène $4100 \mathrm{~K}$

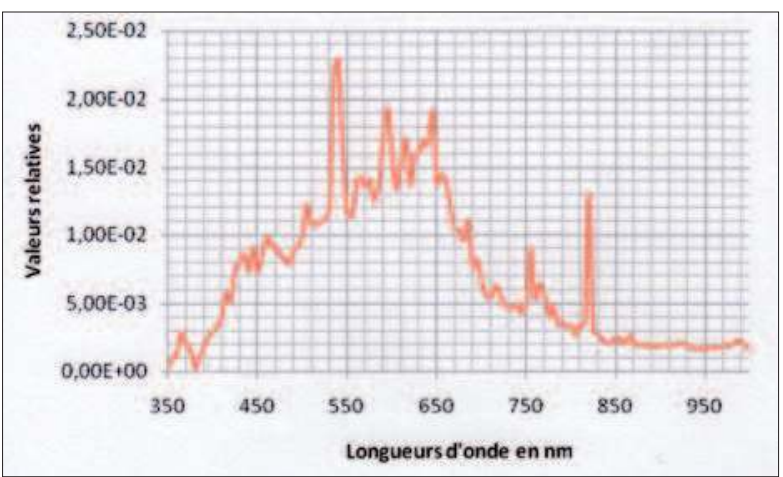

Spectre de la lampe aux iodures métalliques $3900 \mathrm{~K}$ 


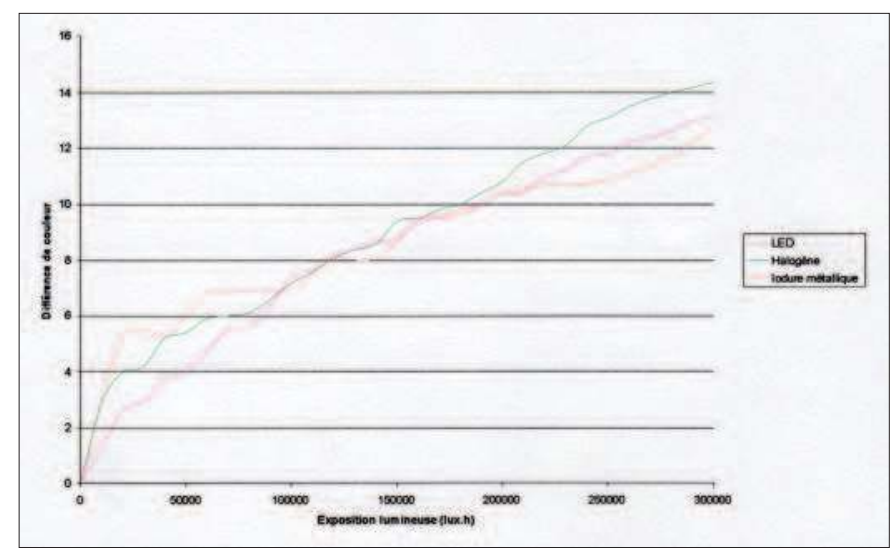

Comparaison après 30 heures d'exposition (300 $000 \mathrm{~lx} / \mathrm{h})$

\section{Analyse des résultats}

Les trois courbes obtenues démontrent bien qu'il y a bien changement de couleur et que la dégradation est semblable quel que soit le type de sources. Pour rappel un $\mathrm{DE}>3$ est visible et nous voyons que dès $50 \mathrm{Klx} / \mathrm{h}$ (soit 50 lux durant 1000 heures !) il est de 4 pour la diode électroluminescente et de 6 pour les deux autres sources. Dès $100 \mathrm{Klx} / \mathrm{h}$, il est de 7 pour toutes les sources puis de 10 au bout de $200 \mathrm{Klx} / \mathrm{h}$. L'argument qu'une source sans ultraviolet assure une protection ne peut être retenu, d'ailleurs toutes les recommandations donnent des valeurs ultraviolets éliminées (10).

\section{Vers le contrôle de l'exposition lumineuse}

Ces deux expérimentations mettent bien en évidence que la dégradation est principalement due à l'action du rayonnement visible et non aux seuls rayons ultraviolets. L'élimination totale des ultraviolets est, pour les cas étudiés, inutile, voire dangereuse car elle donne trop souvent bonne conscience, ce qui évite de se poser trop de questions sur la gestion du rayonnement visible. De même, la recherche de la source idéale me paraît vaine, car ce n'est pas l'absence de rayonnements ultraviolets et infrarouges qui évitera une dégradation c'est aussi, et surtout le rayonnement visible court et moyen qui en est la cause. Il n'y aurait qu'une lumière inactinique, c'est-à-dire une lumière qui n'aurait pas d'effets photochimiques, pour limiter au maximum la dégradation.

Mais en dehors de quelques documents on se voit bien mal réaliser des présentations sous un éclairage rouge! On ne le répétera jamais assez : pour les objets du patrimoine les plus sensibles seul un véritable contrôle du rayonnement visible, ou plutôt, de sa maîtrise, reste la seule et véritable solution pour une meilleure conservation et communication (11). C'est sur ce point, trivial, de la lumière et de l'éclairage (12), que le lien entre la muséographie, le « comment du musée » et la muséologie « le pourquoi » et le «pour qui » du musée, apparaît dans toute sa force. Pour illustrer, quelques mots, sur mon intervention dans l'exposition au musée des Arts décoratifs de Paris.

\section{La demande initiale}

Ce n'est pas le commissaire de l'exposition, mais le conservateur responsable du département de conservation-restauration du musée, Maximilien Durand, qui a fait appel à moi afin que je l'assiste sur cette exposition (Madeleine Vionnet, créatrice de mode), exceptionnelle, par sa durée de sept mois ainsi que par la fragilité des objets présentés soit les robes et accessoires de mode de la collection retrouvée de Madeleine Vionnet.

\section{L'examen du projet d'éclairage}

Rappelons qu'un projet d'éclairage inclut une partie subjective en adéquation avec la scénographie supportant la thématique de l'exposition et une partie objective prenant en compte d'une part la conservation préventive des objets présentés et d'autre part des conditions visuelles confortables pour les visiteurs. La scénographie, éclairage compris, était déjà arrêtée par le cabinet d'Andrée Putmann. Elle se composait d'un environnement noir, brillant et blanc. Un blanc brillant, lumineux et diffus, par un éclairage en contrejour ou par un éclairage zénithal, avec, en plus, l'apport d'un faible éclairage localisé sur chaque mannequin.

L'éclairage général, diffus, provenait des grandes surfaces blanches constituant fonds et plafonds. Pour la réalisation de ces surfaces blanches et translucides les sources de lumière ne pouvaient être que des tubes fluorescents. Le défi pour réaliser ce blanc brillant était que ces surfaces éclairent sans pour autant voir l'image des sources. Pour cela les faisceaux d'éclairement des tubes fluorescents devaient se recouvrir les uns les autres pour disparaître et ne faire apparaître qu'une zone blanche et diffuse.

L'éclairage localisé, éclairage d'accentuation, le sera alors par des projecteurs équipés de lampes halogènes très basse tension avec réflecteur dichroïque. La chaleur des sources étant divisée par deux permet en effet l'emploi de filtres polyester pour travailler le faisceau en teinte et/ou en diffusion. Ces lampes sont pratiquement dépourvues de rayonnement ultraviolet. 


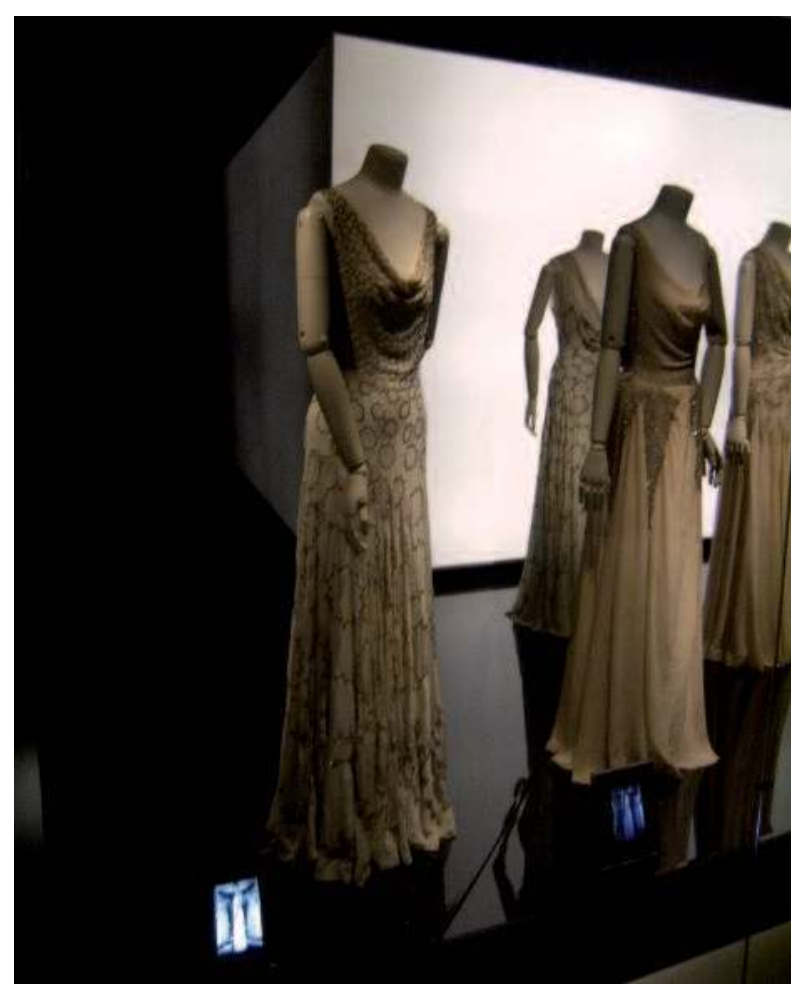

Une exposition lumineuse qui allie conservation préventive et projet scénographique. () J.J. Ezrati

\section{La mission}

Ma mission consistait d'une part à sensibiliser le département des expositions et les concepteurs à l'action de la lumière sur les modèles uniques des robes de la collection particulière de Madeleine Vionnet, et d'autre part à trouver les solutions de conservation préventive, concernant la lumière, respectueuses du projet scénographique. Le dialogue s'est donc engagé avec l'équipe de scénographie sur le choix des sources, la dose de lumière totale à ne pas dépasser, les moyens pour réaliser les objectifs de limitation de l'éclairement, et la mesure de l'exposition lumineuse. De son côté le musée s'est engagé, avec les moyens mis à disposition par le $\mathrm{C} 2 \mathrm{RMF}$, à mesurer l'exposition lumineuse.

\section{L'antagonisme préservation - présentation}

Comme nous le savons la lumière est un des facteurs importants de dégradation des matériaux textiles, qui plus est lorsqu'il s'agit d'une matière comme la soie. Cette fragilité est accrue par les mauvaises conditions de stockage qu'a subies cette collection acquise par les Arts décoratifs qui fait aujourd'hui, après une énorme campagne de restauration, l'objet de cette exposition. Si exposer c'est faire partager un savoir, des connaissances acquises par la recherche, au monde professionnel et à la société toute entière, ce qui est le cas avec cette exposition, c'est aussi mettre en danger cette collection. Le danger vient de toutes parts : la manipulation, le mannequinage, le climat avec ses variations possibles, la poussière et naturellement la lumière. Que puis-je faire avec la lumière, mon domaine d'intervention ? Naturellement réduire l'éclairement à des valeurs minimum ? Dans l'absolu nous répondrons oui pour être conformes à l'enseignement que nous avons reçu en respectant les valeurs recommandées, mais est-ce vraiment cela que nous appellerons de la conservation préventive ? N'est-ce pas oublier que ces actions de prévention «... s'inscrivent dans le contexte ou l'environnement... » (13) et là, la présentation unique, la redécouverte de cette grande dame de la couture française, n'est-elle pas un contexte culturel que nous devons prendre en compte? Naturellement, nous aurons comme objectif «... d'éviter et de minimiser les détériorations ou pertes à venir» (14). À quoi aurait servi de prendre tous les autres risques pour en fin de compte ne pas correctement voir et jouir de ces robes dans leur éclat! Alors que faire pour résoudre cet antagonisme?

\section{Le choix des sources}

Il nous était dicté par le projet d'éclairage : tubes fluorescents pour le rétro-éclairage des surfaces blanches et lampes halogènes pour l'éclairage localisé. Nous avons validé ce choix demandant seulement un filtrage supplémentaire des ultraviolets pour les tubes fluorescents, la possibilité de gradation, et limiter leur nombre au strict nécessaire pour une diffusion parfaite et obtenir la lumière attendue par les concepteurs.

\section{L'exposition lumineuse ou dose totale de lumière à ne pas dépasser}

C'est ici qu'apparaît l'esprit pragmatique de la conservation préventive : les recommandations professionnelles (15) limitent, pour ce type d'objet, l'exposition lumineuse à 15000 lux/h par an, ce qui est l'équivalent d'un éclairage à 75 lux durant un mois. La durée de l'exposition étant de sept mois, nous arrivons à une exposition lumineuse de plus de 110000 lx/h par an soit sept fois la dose admissible et cela avec un éclairement de faible valeur. D'un commun accord nous avons donc fixé la limite de l'exposition lumineuse à 150000 lx/h par an, ou $150 \mathrm{klx} / \mathrm{h}$ par an, soit dix fois la dose admise mais prenant en compte que cette collection, dont le musée est propriétaire, resterait en réserve pour une durée minimum de dix ans (certaines robes, moins fragiles, pourront néanmoins faire partie d'éventuels prêts pendant cette période). 
Les moyens pour réaliser cet objectif

Nous aurions pu limiter l'éclairement à 50 lux, et de fait limiter la dose de moitié, mais là, c'était détruire toute l'esthétique voulue par le scénographe et réduire à néant le plaisir des plus de 60 ans, avec en plus, une forte opposition des concepteurs. Notre solution réside dans un éclairage dynamique (16) variable en intensité lumineuse sous un cycle de temps relativement lent et cela aussi bien pour le rétro éclairage des écrans blancs que pour les projecteurs ponctuels. Les mouvements de variations se faisant sur plusieurs secondes les rendent insensibles aux visiteurs.

\section{Le contrôle}

La mesure de l'exposition lumineuse d'une œuvre exposée sous un éclairage fixe ne pose pas de problème : il suffit de mesurer le niveau d'éclairement à un moment donné puis de multiplier cette valeur par le nombre d'heures d'ouverture de l'exposition. On obtient ainsi l'exposition lumineuse (ou dose totale d'exposition) en lux heures de l'œuvre concernée, pour les reporter sur la fiche de conservation. Lorsque l'éclairage n'est point fixe, mais dynamique, cette méthode n'est plus valable. Il faut donc procéder autrement. Nous avons posé à différents endroits des dosimètres chimiques "LightCheck " (17) de type LCU, faits pour les petites durées d'expositions.

\section{Du bon usage du LightCheck LCU}

Le temps d'exposition est donné théoriquement par la décoloration de la surface sensible passant du bleu au rose. Dans la pratique, il s'avère que cette décoloration ne s'effectue pas de la même manière suivant le type de sources utilisées et il semble plus judicieux de mesurer l'écart de couleur $\triangle$ ECIELAB. Nous avons donc réalisé, aussi bien pour les tubes fluorescents, que pour les lampes halogènes, une décoloration du LightCheck en mesurant l'écart de couleur $\triangle$ ECIELAB à

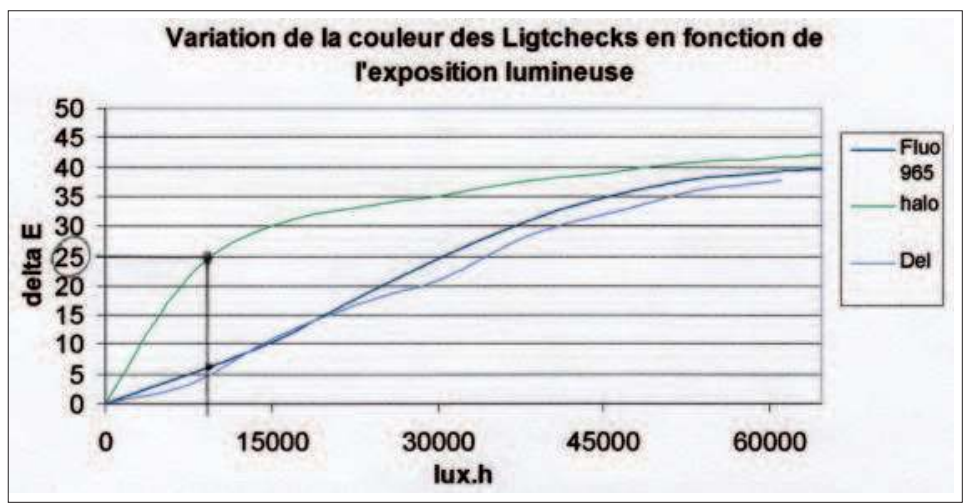

Variation de la couleur des Lightcheck's en fonction de l'exposition lumineuse avec différentes sources intervalles réguliers pour tracer les courbes d'équivalence nous permettant, à partir de l'écart de couleur $\triangle$ ECIELAB obtenu par la mesure, estimer la durée totale d'exposition (DTE).

Dans notre cas, une détérioration équivalente à un $\triangle$ ECIELAB de 25 , sous un éclairage halogène, indique que sous un éclairage fluorescent, pour le même écart de couleur, elle serait de $18 \mathrm{klux} / \mathrm{h}$, soit presque le double, d'où l'importance de l'identification de la source.

\section{Pour conclure}

Que cela soit pour cette dernière démonstration ou les précédentes expérimentations nous en revenons au même point : le danger vient essentiellement du rayonnement visible et c'est celui-ci qu'il faut maîtriser.

\section{Notes}

(1) Brill, T. Light, its interaction with art and antiquities. New-York Plenum, 1980, $287 \mathrm{p}$.

(2) Thomson, G. The museum environment. Londres : Butterworth, 1978, $270 \mathrm{p}$.

(3) May, R. et al. La mise en place d'une politique nationale dans les musées de France : d'une cellule à un département de la conservation préventive, La conservation préventive, une démarche évolutive 1990-2010, Techné, n³4, 2011, pp. 13-24.

(4) Ezrati, J.-J. Protéger le patrimoine des musées contre les ultra-violets, La Lettre de l'OCIM, n², 1988, pp. 8-11.

(5) CIE, Control of damage to museum objects by optical radiation, TR157, Vienne, 2004, $29 \mathrm{p}$

(6) La température de couleur d'une source s'exprime en kelvin (K). D’une manière générale pour une température de $5000 \mathrm{~K}$ on parle d'un blanc neutre, c'est-à-dire que la proportion de rayonnements de grandes longueurs d'onde (rouge) est dans les mêmes proportions que celles des moyennes et courtes radiations (respectivement vertes et bleues) ; au dessus, par exemple $6500 \mathrm{~K}$ il s'agit d'un blanc froid où les radiations de faibles longueurs d'onde sont les plus nombreuses, et inversement pour un blanc chaud à $3000 \mathrm{~K}$.

(7) Le test de la laine ou échelle de la laine bleue est un test reconnu internationalement pour évaluer la solidité d'un textile. Il est constitué d'une échelle de huit échantillons teintés de laine. Le premier (BWS \#1) est le plus sensible, le second (BWS \#2) est deux fois plus résistant que le premier, le troisième (BWS \#3) deux fois plus résistant que le second, et ainsi de suite jusqu'au dernier.

(8) L'espace CIELAB 1976 permet d'inscrire une couleur dans un espace à trois dimensions. Un axe vertical, du noir au blanc appelé L* représentant la clarté allant de 0 à 100, et deux axes horizontaux définissant la chroma, l'axe vert-rouge dénommé $\mathrm{a}^{*}$ et l'axe jaune-bleu dénommé b* Plus les valeurs de $\mathrm{a}^{*}$ et $\mathrm{b}^{*}$ sont fortes $(>40)$ plus la couleur est saturée, et inversement. 
(9) L'écart de couleur, ou différence de couleur est obtenue à partir de la différence des données colorimétriques obtenues par la mesure sur l'échantillon en réserve et sur l'échantillon éclairé. La formule est DE a* ${ }^{*}$ $=\left(\left(\mathrm{L}-\mathrm{L}^{\prime}\right)^{2}+\left(\mathrm{a}^{*}-\mathrm{a}^{* \prime}\right)^{2}+\left(\mathrm{b}^{*}-\mathrm{b}^{* \prime}\right)^{2}\right)-2$.

(10) Voir les documents des notes 2 et 5 en particulier.

(11) Voir l'article et l'ouvrage suivants pour plus de précision sur le contrôle de l'exposition lumineuse : Ezrati, J.-J. Contrôle dynamique d'un réseau de projecteur (CDRP) ou Ezrati, J.-J. L'informatique au service de la conservation, La Lettre de l'OCIM, n 1 1, 1990, pp. 20-23.

(12) Pour rappel, la lumière est un matériau et l'éclairage en est sa maîtrise à des fins définies comme dans notre domaine, réduire ses effets sur la matière, permettre une vision correcte, quel que soit l'âge, tout en participant à la mise en espace du contenu comme élément de la scénographie. Voir, Ezrati, J.-J. L'éclairage muséographique, La Lettre de l'OCIM, n95, 2004, pp. 31-34 et Ezrati, J.-J. Théorie, technique et technologie de l'éclairage muséographique. Nantes : Éditions AS, 2002, 143 p.
(13) (14) (15) Terminologie de la conservation-restauration du patrimoine culturel matériel, définition de la conservation préventive, résolution adoptée par les membres de l'ICOM-CC à l'occasion de la XVe Conférence Triennale, New Delhi, 22-26 septembre 2008.

(16) Ezrati, J.-J. Approche pragmatique de la conservation préventive : l'accompagnement à la conception lumière de l'exposition Madeleine Vionnet au musée des Arts Décoratifs de Paris du ler août 2009 au ler février 2010. Préprint de la conférence d'ICOM CC, Lisbonne.

(17) Dupont, A.-L., Cucci, C., Loisel, C., Bacci, M. et Lavédrine, B. Development of LightCheck ${ }^{\circledR}$ Ultra, a novel dosimeter for monitoring lighting conditions of highly photosensitive artefacts in museums, Studies in Conservation n53, 2008, pp. 49-72. 